\title{
Chaplygin Gas of Tachyon Nature Imposed by Noether Symmetry and Constrained via $H(z)$ Data
}

\author{
L. G. Collode ${ }^{*}$ and G. M. Kremen \\ Departamento de Física, Universidade Federal do Paraná, 81531-980 Curitiba, Brazil
}

(Dated:)

\begin{abstract}
An action of general form is proposed for a Universe containing matter, radiation and dark energy. The latter is interpreted as a tachyon field non-minimally coupled to the scalar curvature. The Palatini approach is used when varying the action so the connection is given by a more generic form. Both the self-interaction potential and the non-minimally coupling function are obtained by constraining the system to present invariability under global point transformation of the fields (Noether Symmetry). The only possible solution is shown to be that of minimal coupling and constant potential (Chaplygin gas). The behavior of the dynamical properties of the system is compared to recent observational data, which infers that the tachyon field must indeed be dynamical.
\end{abstract}

PACS numbers: 98.80.-k, 98.80.jk

* lgcollodel@gmail.com

† kremer@fisica.ufpr.br 


\section{INTRODUCTION}

Tachyons have been vastly studied in M/String theories. Since the realization of its condensation proprieties, researchers have gained interest in its applications in cosmology. At first, there was the problem of describing the string theory tachyon by an effective field theory that would lead to the correct lagrangian in classical gravity. The first classical description of the tachyon field $([1,2])$ addressed the lagrangian problem, making way for building the first model within tachyon cosmology ([3]).

Being a special kind of a scalar field, it present negative pressure, making the tachyon a natural candidate to explain dark energy ([4 - 7$]$ ). The inflationary period could also be explained if one considers the inflaton to behave as a tachyon field. Many different attempts were made under this assumption, testing a wide variety of self-interacting potentials such as power-laws, exponentials and hyperbolic functions of the field ( 8 [15]). The possible case scenario where the tachyon plays both roles, inflaton and dark energy, has also been studied in the works ([16, 17]), where the first establishes constraints on the potential so the radiation's era could commence.

The studies above introduced a tachyon field which is minimally coupled through the metric, hence providing just another source for the gravitational field. Nevertheless, such fields might also be considered to be non-minimally coupled to the scalar curvature, becoming part of the spacetime geometry by generating a new degree of freedom for gravity. In this scenario, the gravitational constant $G$ becomes a variable function of spacetime.

Tachyon fields in the non-minimal coupling context were analyzed for both the inflationary period ([18]) and the current era ([19]). In those cases, the coupling functions and the self-interacting potentials were given in an $a d-h o c$ manner, as exponentials and power-law forms.

Every time we choose a different coupling or potential function, we create a new cosmological model, or even a new theory of gravity in the non-minimal case. This is a very difficult task since the lack of experiments and observations obligates one to find heuristics arguments to support the choice made. The advantages of searching for symmetries in systems where the lagrangian is known is widely entertained, it not only helps us find exact solutions but might also give us physically meaningful constants of movement. What is less appreciated is the fact that one can constrain a system (one that lacks a closed form of the functional) to present symmetry. In what concerns non-minimally coupled tachyon fields, Noether symmetries were used to establish the coupling and self-interaction functions in the papers $([20,21])$. The latter makes use of the Palatini approach, in a way to generalize the theory, since the non-minimal coupling can provide a metric-independent connection.

The Chaplygin gas was first introduced by Chaplygin in 1902 ([22]) in the realms of aerodynamics. This gas features an exotic equation of state $\left(p_{c} \propto-1 / \rho_{c}\right)$, which was originally used to describe the lifting force on a wing of an airplane. Because its pressure is negative, the Chaplygin gas became a good candidate to explain dark energy (23 28]). The attempts to correlate fields and fluids soon showed that the constant potential tachyon field behaves as a Chaplygin gas ([29-32]). Its equation of state allows generalizations, giving rise to the so called Generalized Chaplygin Gas, or just GCG. This gas exerts a negative pressure proportional in moduli to the inverse of some power of its energy density and was investigated in works such as $([33] 37)$, including its relationship to a - now, not constant potential - tachyon field ([38]). Originally, the equation of state of a Chaplyigin gas was so simple that even with the exhausted studies about the GCG there was still plenty of room for further generalizations. Endowing the EoS with a linear barotropic term, which alone would describe an ordinary fluid, enriched the GCG which under this assumption is called the Modified Chaplygin Gas, MCG. Its motivations lie precisily on the possible field nature of the gas ([39]), and its parameters have been constrained via observational analysis ([40]). Further generalizations account for higher order energy density terms in the EoS of a Chaplygin Gas, the Extended Chaplyigin Gas, ECG ([41, 42]).

In this work, we start from a very general lagrangian for a tachyon field non-minimally coupled to the scalar curvature. Matter and radiation fields are also included in the system as perfect fluids from the beginning. The connection is initially taken to be metric independent and the action is also varied with respect to it, a process known as the Palatini approach. Since we consider a flat, homogeneous and isotropic Universe, the flat FriedmannLemaître-Robertson-Walker (FLRW) metric is used to rewrite our functional in the form of a point-like lagrangian. This presents an extra term than usual, which comes from the independent connection. The system is constrained to that one which presents invariance under continuous point transformations, or a Noether symmetry. The coupling and self-interaction potential functions of the tachyon field are then determined. Every new field added to the lagrangian clearly influences these point transformations. For that matter, it is important to start off from a complete system (including the radiation fields) if one takes symmetry as a first principle. We show that for this system to be Noether symmetrical, the non-minimal coupling must vanish and the self-interaction potential must be constant, hence the tachyonic Chaplygin gas. The system is initially composed of five free parameters, namely the Hubble constant, the three density parameters for recent times and the normalized constant potential. The radiation parameter is then established in a $a d$-hoc way so we are left with four different free parameters. These are determined via the $\chi^{2}$ analysis for the recent $H(z)$ data from SNe and gamma-ray bursts. We show that although dark energy tends asymptotically to a cosmological constant, any small discrepancies make the tachyon field dynamically active, so the Chaplygin gas 
presents property of transition from pressureless matter to dark energy.

In order to clarify the typos and notations here used, we remark: the metric signature is $(+,-,-,-)$; the LeviCivita connection is written with a tilde, $\tilde{\Gamma}_{\mu \nu}^{\lambda}=\left\{\begin{array}{l}\lambda \\ \mu \nu\end{array}\right\}$ while the independent connection is given without it $\Gamma_{\mu \nu}^{\lambda}$. Natural constants were rescaled to the unity $(8 \pi G=c=1)$. Throughout the whole paper, derivatives in equations are presented as follows: dots represent time derivatives, while $\partial_{q^{i}} \equiv \frac{\partial}{\partial q^{i}}$ and $\partial_{\dot{q}^{i}} \equiv \frac{\partial}{\partial \dot{q}^{i}}$ stand for partial derivatives with respect to the generalized coordinate $q^{i}$ and velocity $\dot{q}^{i}$, respectively.

\section{ACTION AND POINT LAGRAGIAN}

A generalization of the general theory of relativity is proposed through a non-minimal coupling of a function of the tachyon field. The general action for both geometry and source is written

$$
S=\int d^{4} x \sqrt{-g} f(\phi) R-V(\phi) \sqrt{1-\partial_{\mu} \phi \partial^{\mu} \phi}-\mathcal{L}_{s},
$$

where $\phi$ is the tachyon field, $f(\phi)$ is the non-minimal coupling function, $V(\phi)$ is the self-interaction potential and $\mathcal{L}_{s}$ is the lagrangian density of other sources (matter and radiation).

In order to attain a more general theory we allow the connection to be metric independent. The variation of the action with respect to the connection $\Gamma_{\mu \nu}^{\rho}$ results in the well-known form

$$
\Gamma_{\mu \nu}^{\rho}=\tilde{\Gamma}_{\mu \nu}^{\rho}+\frac{1}{2 f}\left(\delta_{\nu}^{\rho} \partial_{\mu} f+\delta_{\mu}^{\rho} \partial_{\nu} f-g_{\mu \nu} \partial^{\rho} f\right)
$$

where $\tilde{\Gamma}_{\mu \nu}^{\rho}$ is the Levi-Civita connection.

Usually, the self-interaction potential and the coupling function are set in a ad-hoc manner. Instead of approaching the problem this way, we would like to constrain the system to that which has a Noether symmetry. This is done by operating a variational vector field on the point-like lagrangian, and for this, we need to rewrite it on a specific metric. For a flat, homogeneous and isotropic Universe, spacetime is described by the flat FLRW metric. The point-like functional in (1) then becomes

$$
L=6 f\left(\ddot{a} a^{2}+\dot{a}^{2} a\right)-\frac{3 a^{3}}{2 f}\left(\partial_{\phi} f \dot{\phi}\right)^{2}+3 a^{3} \partial_{\phi}^{2} f \dot{\phi}^{2}+3 a^{3} \partial_{\phi} f \ddot{\phi}+9 \dot{a} a^{2} \partial_{\phi} f \dot{\phi}-a^{3} V \sqrt{1-\dot{\phi}^{2}}-a^{3} \rho_{s},
$$

and $\rho_{s}$ is point-like lagrangian for a perfect fluid $([\underline{43}])$.

In this system, besides dark energy, the Universe is composed by matter (ordinary and dark) and radiation. Both dark matter and ordinary matter are treated as dust, hence represented by the same entity here. As the Universe expands, matter's density decrease with $a^{-3}$ while radiation's with $a^{-4}$. The lagrangian above contains second-order terms which are more tedious to deal with. Since the action limits are fixed, we can integrate these terms by parts, without loss of generality, so we can work with a first-order lagrangian, which reads

$$
L=-6 f \dot{a}^{2} a-6 a^{2} \dot{a} \partial_{\phi} f \dot{\phi}-a^{3} V \sqrt{1-\dot{\phi}^{2}}-\frac{3 a^{3}}{2 f}\left(\partial_{\phi} F \dot{\phi}\right)^{2}-\rho_{m}^{0}-\frac{\rho_{r}^{0}}{a},
$$

where $\rho_{m}^{0}$ and $\rho_{r}^{0}$ are the recent values of the total density of matter and radiation, respectively, in the Universe.

\section{NOETHER SYMMETRIES}

Our system may now be constrained to that which is endowed with a Noether symmetry by finding the forms of $f(\phi)$ and $V(\phi)$ that allow symmetrical point transformation. This means that our lagrangian shall have such a form that a specific continuous transformation of the generalized coordinates $a \rightarrow \bar{a}$ and $\phi \rightarrow \bar{\phi}$ preserves the general form of the functional,

$$
L(\bar{a}, \bar{\phi})=L(a, \phi) .
$$

In order to find the function forms of $V(\phi)$ and $f(\phi)$ that allow such transformation, we need to apply a certain vector field on the lagrangian (4). This vector field, $\mathbf{X}$, is then called a variational symmetry, or complete lift, and reads

$$
\mathbf{X} \equiv \alpha^{i} \partial_{q^{i}}+\dot{\alpha}^{i} \partial_{\dot{q}^{i}}
$$


where the coefficients $\alpha^{i}$ are functions of the generalized coordinates $a, \phi$. The operation of $\mathbf{X}$ on the lagrangian is simply the Lie derivative of $L$ along this vector field $\left(L_{\mathbf{X}} L\right)$. According to the Noether theorem, if this derivative vanishes, there will be a conserved quantity named Noether charge. Hence, this will be a variational symmetry if

$$
\mathbf{X} L=L_{\mathbf{X}} L=0,
$$

such that

$$
L_{\Delta}\left\langle\theta_{L}, \mathbf{X}\right\rangle=0
$$

where $\Delta=d / d t$ is the dynamical vector field and

$$
\theta_{L}=\frac{\partial L}{\partial \dot{q}^{i}} d q^{i}
$$

is the locally defined Cartan one-form. The brackets represent the scalar product between vector field and one-form, in the Dirac notation. Thus, the Noether charge reads

$$
\Sigma_{0} \equiv\left\langle\theta_{L}, \mathbf{X}\right\rangle=\alpha^{i} \frac{\partial L}{\partial \dot{q}^{i}} .
$$

The condition (7) reads in full form,

$$
\begin{aligned}
0 & =X L \\
& =\alpha \partial_{a} L+\beta \partial_{\phi} L+\left(\dot{a} \partial_{a} \alpha+\dot{\phi} \partial_{\phi} \alpha\right) \partial_{\dot{a}} L+\left(\dot{a} \partial_{a} \beta+\dot{\phi} \partial_{\phi} \beta\right) \partial_{\dot{\phi}} L,
\end{aligned}
$$

which for our system becomes

$$
\begin{aligned}
0 & =\alpha\left(-6 f \dot{a}^{2}-12 a \dot{a} \partial_{\phi} f \dot{\phi}-3 a^{2} V \sqrt{1-\dot{\phi}^{2}}-\frac{9 a^{2}\left(\partial_{\phi} f\right)^{2} \dot{\phi}^{2}}{2 f}+\frac{\rho_{r}^{0}}{a^{2}}\right) \\
& +\beta\left(-6 \partial_{\phi} f \dot{a}^{2} a-6 a^{2} \dot{a} \partial_{\phi}^{2} f \dot{\phi}-a^{3} \partial_{\phi} V \sqrt{1-\dot{\phi}^{2}}+\frac{3 a^{3}\left(\partial_{\phi} f\right)^{3} \dot{\phi}^{2}}{2 f^{2}}-\frac{3 a^{3} \partial_{\phi} f \partial_{\phi}^{2} f \dot{\phi}^{2}}{f}\right) \\
& +\left(\partial_{a} \alpha \dot{a}+\partial_{\phi} \alpha \dot{\phi}\right)\left(-12 f \dot{a} a-6 a^{2} \partial_{\phi} f \dot{\phi}\right)+\left(\partial_{a} \beta \dot{a}+\partial_{\phi} \beta \dot{\phi}\right) \\
& \times\left(-6 a^{2} \dot{a} \partial_{\phi} f+\frac{a^{3} V \dot{\phi}}{\sqrt{1-\dot{\phi}^{2}}}-3 a^{3} \frac{\left(\partial_{\phi} f\right)^{2} \dot{\phi}}{f}\right)
\end{aligned}
$$

where $\alpha=\alpha^{1}$ and $\beta=\alpha^{2}$.

The equation above must hold for any value of $\dot{a}$ and $\dot{\phi}$. If it were a polynomial equation for these dynamical variables one could simply make all coefficients equal to zero, but the different powers of the square roots turn the task a little more complicated. We shall differentiate with respect to these quantities and evaluate the resulting equations for different values of $\dot{a}$ and $\dot{\phi}$, then we get the solutions for $\alpha(a, \phi), \beta(a, \phi), V(\phi)$ and $f(\phi)$.

Making $\dot{a}=\dot{\phi}=0$ in (12) we get

$$
3 \alpha a^{2} V-\alpha \frac{\rho_{r}^{0}}{a^{2}}+\beta a^{3} \partial_{\phi} V=0
$$

Differentiating equation (12) three times with respect to $\dot{\phi}$ and evaluating at $\dot{\phi}=0$ and $\dot{a}=1$ gives

$$
3 a^{3} V \partial_{a} \beta=0,
$$

hence $\beta \neq \beta(a)$. Similarly, differantitating (12) once with respect to $\dot{\phi}$, multiplying it by $\left(1-\dot{\phi}^{2}\right)^{3 / 2}$ and evaluating at $\dot{\phi}=1$ and $\dot{a}=0$ we get

$$
V \partial_{\phi} \beta=0,
$$


and we conclude that $\beta=\beta_{0}$ is a constant, since the potential must be non-zero. The fourth derivative of (12) with respect to $\dot{\phi}$, evaluated at $\dot{\phi}=0$ and taking into account that $\partial_{\phi} \beta=0$ leads to

$$
9 \alpha a^{2} V+3 \beta a^{3} \partial_{\phi} V=0
$$

Since $\rho_{r}^{0} \neq 0$, dividing (16) by 3 and equating with (13) results in $\alpha=0$ and $V=V_{0}$, constant potential. Thus, equation (12) reduces to

$$
-6 \partial_{\phi} f \dot{a}^{2} a-6 a^{2} \dot{a} \partial_{\phi}^{2} f \dot{\phi}+\frac{3 a^{3}\left(\partial_{\phi} f\right)^{3} \dot{\phi}^{2}}{2 f^{2}}-\frac{3 a^{3} \partial_{\phi} f \partial_{\phi}^{2} f \dot{\phi}^{2}}{f}=0
$$

and it is clear that $\partial_{\phi} f=0$. The coupling must then be minimal.

\section{EQUATIONS OF MOTION}

The lagrangian (4), for constant self-interaction potential and $f=1 / 2$ (to regain Einstein's constant according to the notation adopted), reads

$$
L=-3 a \dot{a}^{2}-V_{0} a^{3} \sqrt{1-\dot{\phi}^{2}}-\rho_{m}^{0}-\frac{\rho_{r}^{0}}{a} .
$$

The Friedmann equation is obtained through the energy equation $E_{L}=\dot{a} \frac{\partial L}{\partial \dot{a}}+\dot{\phi} \frac{\partial L}{\partial \dot{\phi}}-L$, which gives

$$
H^{2}=\frac{1}{3} \rho
$$

where $H=\dot{a} / a$ is the Hubble parameter and $\rho=\rho_{m}+\rho_{r}+\rho_{\phi}$ is the total energy density of the fields, being

$$
\rho_{\phi}=\frac{V_{0}}{\sqrt{1-\dot{\phi}^{2}}}
$$

the energy density of the tachyon field, $\rho_{m}=\rho_{m}^{0} / a^{3}$ and $\rho_{r}=\rho_{r}^{0} / a^{4}$ the matter's and the relativistic material's densities, respectively.

The Euler-Lagrange equation for the scalar factor, together with (19), provides the acceleration equation, being

$$
\frac{\ddot{a}}{a}=-\frac{1}{6}(\rho+3 p)
$$

where $p=p_{r}+p_{\phi}$ is the pressure of the fields (as usual, matter behaves as dust so $p_{m}=0$ ), and $p_{r}=\rho_{r} / 3$. The pressure exerted by the tachyon field is

$$
p_{\phi}=-V_{0} \sqrt{1-\dot{\phi}^{2}}
$$

The Euler-Lagrange equation for the tachyon field gives the generalized Klein-Gordon equation for the field, which is the same as the fluid equation for dark energy when written in terms of its energy density and pressure

$$
\dot{\rho}_{\phi}+3 H\left(\rho_{\phi}+p_{\phi}\right)=0
$$

An equation of state in the form $p(\rho)$ can now be written for the tachyon field. From equation (20) we see that $\sqrt{1-\dot{\phi}^{2}}=V_{0} / \rho_{\phi}$, which when substituted in (22) yields

$$
p_{\phi}=-\frac{V_{0}^{2}}{\rho_{\phi}} .
$$

The Chaplygin gas is a fluid described by an equation of state of the kind

$$
p=-\frac{A}{\rho}
$$

where $A$ is a positive defined constant, which is precisely the same as (24) for $A=V_{0}^{2}$. Thus, as widely know from the literature, see e.g. ([29 32] ), a tachyon field only minimally coupled to the scalar curvature, of constant potential, behaves as a Chaplygin gas. 


\section{NOETHER CONSTANT}

Any lagrangian system endowed with a Noether symmetry will present a constant of motion, as stated by Noether's theorem. The Noether charge (10) here becomes

$$
\begin{aligned}
\Sigma_{0} & =\alpha \frac{\partial L}{\partial \dot{a}}+\beta \frac{\partial L}{\partial \dot{\phi}} \\
& =\frac{V_{0} a^{3} \dot{\phi}}{\sqrt{1-\dot{\phi}^{2}}},
\end{aligned}
$$

which is simply the first integral of (23).

\section{SOLUTIONS}

The energy density of the Chaplygin gas, and its pressure, can be rewritten as functions of the scale factor, using equation (10). These forms are well known from literature and read

$$
\rho_{\phi}=\sqrt{\frac{\Sigma_{0}^{2}}{a^{6}}+V_{0}^{2}} ; \quad p_{\phi}=-\frac{V_{0}^{2}}{\sqrt{\frac{\Sigma_{0}^{2}}{a^{6}}+V_{0}^{2}}},
$$

where $\Sigma_{0}$ is the Noether constant. From this equation, we see the dual nature of the Chaplygin gas, which behaves as dust matter for $a \leq 1$

$$
\rho_{\phi} \sim \frac{\Sigma_{0}}{a^{3}} ; \quad p_{\phi} \sim 0
$$

and as a cosmological constant for $a \geq 1$

$$
\rho_{\phi} \sim V_{0} ; \quad p_{\phi} \sim-V_{0}
$$

In order to obtain our parameters' curves with respect to the redshift, we use the relationship $a=1 /(1+z)$. The Friedmann equation (19) then becomes

$$
H^{2}=\frac{1}{3}\left(\sqrt{\Sigma_{0}^{2}(1+z)^{6}+V_{0}^{2}}+\rho_{m}^{0}(1+z)^{3}+\rho_{r}^{0}(1+z)^{4}\right) .
$$

The equation above can be written in a dimensionless form by dividing it by the Hubble constant, $H_{0}^{2} \equiv H(0)^{2}=$ $\rho^{0} / 3$, where $\rho^{0}$ is the current density of all fluids in the Universe, giving

$$
\frac{H^{2}}{H_{0}^{2}}=\left(\sqrt{\bar{\Sigma}_{0}^{2}(1+z)^{6}+\bar{V}_{0}^{2}}+\Omega_{m}^{0}(1+z)^{3}+\Omega_{r}^{0}(1+z)^{4}\right),
$$

where $\Omega_{i}^{0} \equiv \rho_{i}^{0} / \rho^{0}$ is the current density parameter of the $i$-th component. The bars indicate that the constants have also been divided by the current density, i.e., $\bar{\Sigma}_{0}=\Sigma_{0} / \rho^{0}$ and $\bar{V}_{0}=V_{0} / \rho^{0}$, then the density parameter for the Chaplygin gas is simply

$$
\Omega_{\phi}^{0}=\sqrt{\bar{\Sigma}_{0}^{2}+\bar{V}_{0}^{2}} .
$$

This last relationship allows us to investigate the evolution of the Hubble parameter in terms of dark energy's density parameter, instead of the Noether charge. Finally, we write

$$
H=H_{0}\left[\sqrt{\left[\left(\Omega_{\phi}^{0}\right)^{2}-\bar{V}_{0}^{2}\right](1+z)^{6}+\bar{V}_{0}^{2}}+\Omega_{m}^{0}(1+z)^{3}+\Omega_{r}^{0}(1+z)^{4}\right]^{1 / 2} .
$$

Recent observations $([44,45])$ limit the range of values associated to these parameters. In particular, there is great confidence that $\Omega_{r}^{0} \sim 8,5 \times 10^{-5}$, so we may adopt this result but will constrain the four remaining parameters (namely $H_{0}, \Omega_{m}^{0}, \Omega_{\phi}^{o}$, and $\left.\bar{V}_{0}\right)$ via $H(z)$ data. 


\begin{tabular}{|ccc|}
\hline \hline $\mathrm{z}$ & $H_{\text {obs }}$ & $\sigma$ \\
\hline 0.07 & 69 & 19.6 \\
\hline 0.09 & 69 & 12 \\
\hline 0.12 & 68.6 & 26.2 \\
\hline 0.17 & 83 & 8 \\
\hline 0.179 & 75 & 4 \\
\hline 0.199 & 75 & 5 \\
\hline 0.2 & 72.9 & 29.6 \\
\hline 0.24 & 79.69 & 3.32 \\
\hline 0.27 & 77 & 14 \\
\hline 0.28 & 88.8 & 36.6 \\
\hline 0.352 & 83 & 14 \\
\hline 0.4 & 95 & 17 \\
\hline 0.43 & 86.45 & 3.27 \\
\hline 0.48 & 97 & 62 \\
\hline 0.593 & 104 & 13 \\
\hline 0.68 & 92 & 8 \\
\hline 0.781 & 105 & 12 \\
\hline 0.875 & 125 & 17 \\
\hline 0.88 & 90 & 40 \\
\hline 0.9 & 117 & 23 \\
\hline 1.037 & 154 & 20 \\
\hline 1.3 & 168 & 17 \\
\hline 1.43 & 177 & 18 \\
\hline 1.53 & 140 & 14 \\
\hline 1.75 & 202 & 40 \\
\hline \hline & & \\
\hline
\end{tabular}

TABLE I. Observational values for $H(z)$ and their respective errors ([46 50$])$.

Table [1 presents 25 measurements of the Hubble parameter from SNe and gamma-ray burst (46 50$]$ ). The function built for the Hubble parameter (33) depends on the redshift, plus four different parameters. Hence, $H=H\left(z, H_{0}, \Omega_{m}^{0}, \Omega_{\phi}^{0}, \bar{V}_{0}\right)$. The values assumed by these parameters that best fit the observational data are the ones the minimize the function

$$
\chi^{2}=\sum_{i=1}^{25}\left[\frac{H_{o b s}\left(z_{i}\right)-H\left(z_{i}, H_{0}, \Omega_{m}^{0}, \Omega_{\phi}^{0}, \bar{V}_{0}\right)}{\sigma_{i}}\right]^{2} .
$$

A primary condition for a good fit is that $\chi^{2} / d o f \leq 1$, where dof stands for degrees of freedom and in this case is given by the number of data points, $d o f=25$. Our minimized $\chi^{2}$ is given by $H_{0}=69.6524, \Omega_{m}^{0}=0.288261, \Omega_{\phi}^{0}=$ 0.711654 and $\bar{V}_{0}=0.709957$ resulting in $\chi^{2}=12.8676$ and $\chi^{2} /$ dof $=0.507504$. Marginalizing over two parameters allows us to analyze the correlation of the remaining by plotting the contours of their distributions within some confidence interval.

The correlation between dark energy and matter density parameter is strong. The $H(z)$ does not seem to impose very strict constraints for our current matter density, but for dark energy we see that within 3- $\sigma$ all points lie in the range $0.697 \leq \Omega_{\phi}^{0} \leq 0.726$, Fig 1 . The correlation between $\Omega_{\phi}^{0}$ and $\bar{V}_{0}$ is much stronger, as one would expect since the potential defines the energy density. Nevertheless, it is interesting enough to see the form these ellipses take in Fig. 2 whilst the current density parameter for dark energy is given by (32). The case $\Omega_{\phi}^{0}=\bar{V}_{0}$ is just the cosmological constant scenario. From the figure, we see very thin ellipses with a slope close to the unity. The best fit parameters listed above show a very small difference between the two of them, and as we will see this difference grows bigger in the past, but there is a high tendency for the cosmological constant.

The evolution of the density parameters for different redshift scales are shown below. In Fig. 3 3 radiation is neglected for its energy density is too small to be observed. As the redshift increases dark energy falls but ever more slowly, and for values $z \geq 2$ its density decreases so smoothly that it almost appears to be constant. This is due to the small 


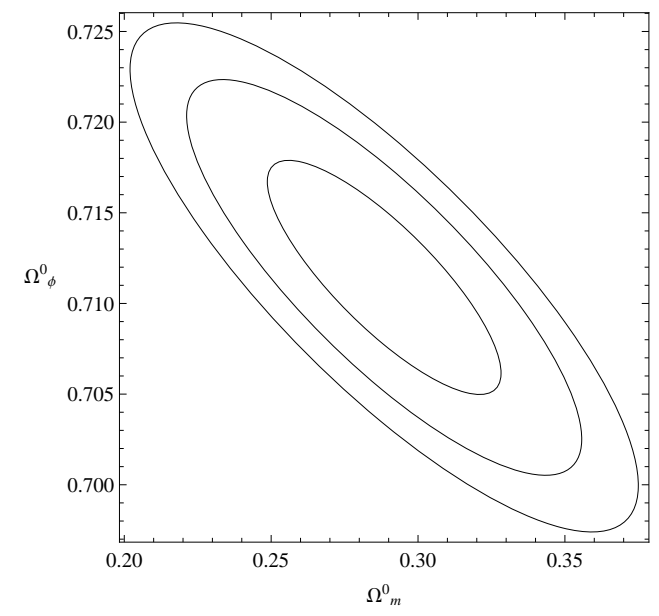

FIG. 1. Confidence intervals for 1-,2- and $3-\sigma$ for the density parameters $\Omega_{m}^{0}$ and $\Omega_{\phi}^{0}$.

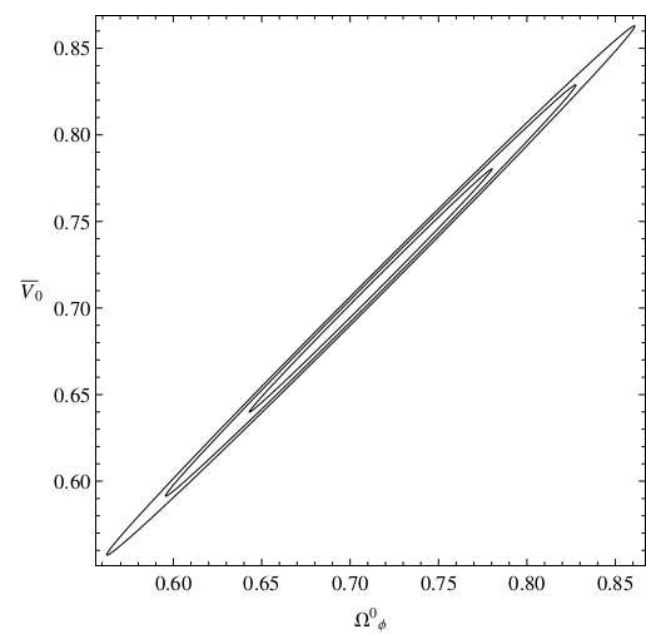

FIG. 2. Confidence intervals for 1-,2- and 3- $\sigma$ for the density parameter $\Omega_{\phi}^{0}$ and the constant potential $\bar{V}_{0}$.

difference between $\Omega_{\phi}^{0}$ and $\bar{V}_{0}$ that makes the tachyon field dynamical and the Chaplygin gas property thrive, from equations (27) and (28) it becomes clear that dark energy decays into matter fields as the redshift grows and the term $\bar{\Sigma}_{0}$ outpaces $\bar{V}_{0}$. Furthermore, we are now looking at the matter era, hence the almost constat behavior. In Fig. 4 we see the evolution of the density parameters for radiation and the combination of the Chaplygin gas and matter, since the first behaves as the latter. In this scenario, the densities equality happen a bit earlier in out history than expected from the cosmological constant case. For instance, we have $z_{e q}=3968.15$ (approximately 37.5 thousands years since the beginning of the Universe) whereas for a non-dynamical field describing we would expect $z_{e q} \sim 3600$ (about 47 thousand years old). Although a transition happening at higher redshifts does not influence the time at which recombination occurs (once it depends on the temperature), the sooner increase in dark matter's energy density allows it to combine and form potential wells earlier, giving room for structure formation, some of which we have recently discovered and turned out to be quite old $([51,52])$.

The ratio $\omega_{\phi}=p_{\phi} / \rho_{\phi}$, between dark energy's pressure and energy density is show in Fig. 5. As expected from equation (27), the ratio tends asymptotically to -1 as the Universe expands but approaches zero quickly as the redshift increases, when dark energy finally becomes a pressureless field. It becomes clearer the role of the Chaplygin gas even though we are approaching the cosmological constant in recent times.

The deceleration parameter $q$ is plotted in Fig. 6. The transition from a decelerated to an accelerated expansion happens at $z_{t}=0.65$, while for our current time $q_{0}=-0.56$, both results in agreement with observations ([53]).

Although the Chaplygin gas has been extensively studied before, new observational data provides great motivation to revisit the model and set new constraints. The evolution of the Hubble parameter described by this model, together with the data we used to define our parameters is shown in Fig. 7. Unfortunately, there are not satisfactorily 


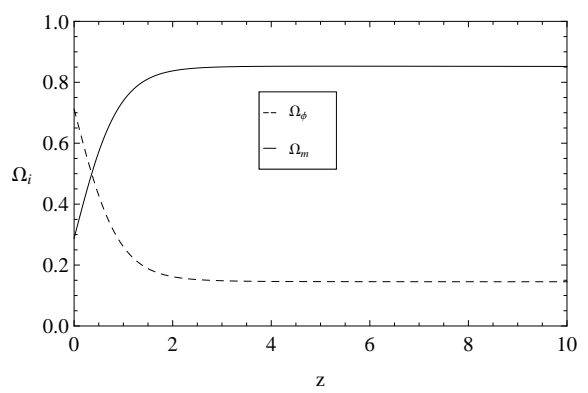

FIG. 3. The dotted lines stand for dark energy while the solid lines represent dark matter. As we enter the matter dominated era, dark energy decays into matter fields contributing even more for its dominance, with its energy density falling ever more slowly, assuming an almost constant behavior.

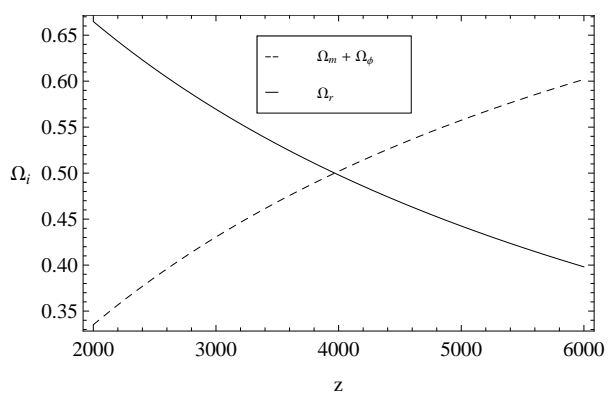

FIG. 4. Density parameters plotted for high redshift values. The dashed line represent the matter fields, where the Chaplygin gas is included once it behaves as dust at this point. Solid line stand for radiation's energy density parameter. Equality in densities happen at $z=3968.15$.

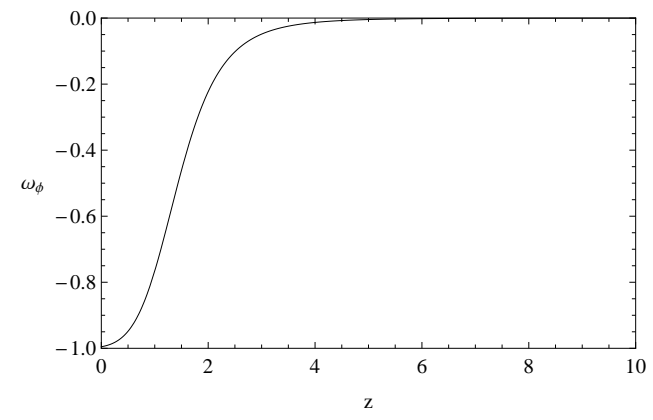

FIG. 5. Ratio between pressure and energy density for the Chaplygin gas. Any small difference between $\Omega_{\phi}^{0}$ and $\bar{V}_{0}$ grows considerably with the redshift and dark energy eventually becomes a pressureless field, hence matter.

many measurements to make solid statistics for this parameter as there are for the distance modulus, for instance. Also, the errors associated with the data from gamma-ray bursts are much bigger than one would desire them to be. Nevertheless, these sources provide information from a much younger Universe compared to SNe, making it worthwhile for testing models and constraining parameters.

\section{CONCLUSIONS}

In this work, we started from a general action where a tachyon field represents the nature of dark energy. We allowed it to be non-minimally coupled to the scalar curvature and we considered the connection to be independent through the Palatini approach. Dark matter, baryonic matter and relativistic material were included in source fields, as our intention was to build a more complete model. Instead of establishing the self-interaction potential and the coupling function in a $a d-h o c$ manner, we stated that symmetry should play a more primary role and only functions capable 


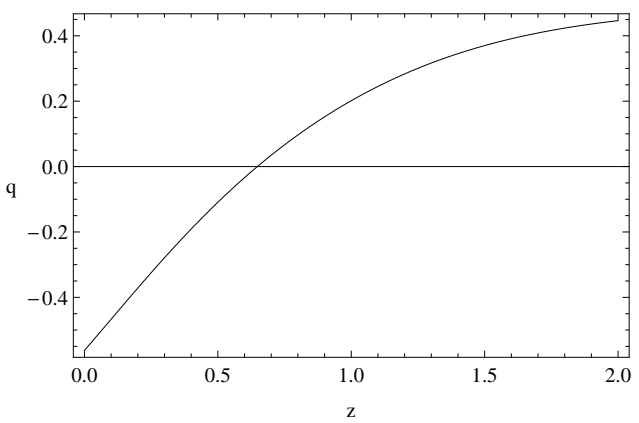

FIG. 6. Deceleration parameter. Expansion becomes accelerated at $z_{t}=0.65$ and the current value of this parameters stands at $q_{0}=-0.56$.

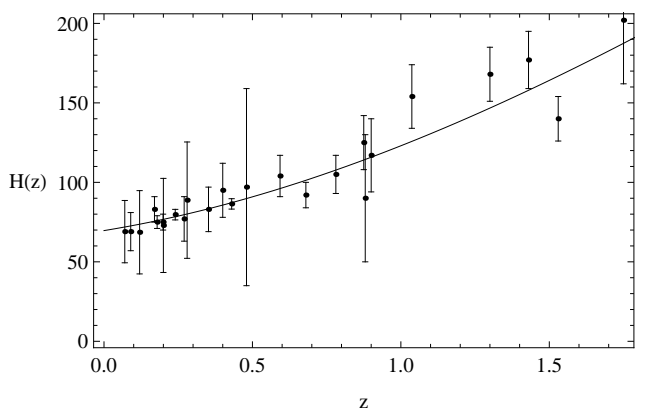

FIG. 7. The observational data for the Hubble parameter ( 46 [50 $]$ ) favor the dynamical field over the cosmological constant. The more dynamical the tachyon field is, greater is the inclination of the $H(z)$ curve. Even with such big error bars, we can identify the case $\bar{V}_{0}=0.69$ as being the one that accommodates the more data.

of composing a continuous and symmetric point transformation on the generalized coordinates would be considered. This lead to the simpler case where the tachyon field is only minimally coupled and its potential is constant, behaving as a Chaplygin gas.

The theoretical framework of the Chaplygin gas has been deeply investigated and is widely found in the literature. For this reason, we focused on more recent observational data to constrain the dynamics of the system. The Hubble parameter suggests that, be the Chaplygin gas the underlying nature of dark energy, it shall be slightly dynamical, as opposed to its cosmological constant particular case since, as we see, any small difference between its constant potential and current density parameter grows considerably with the redshift. As this component presents a dual behavior, acting as dark energy for small redshifts and decaying into matter fields later on, the matter era begins earlier in the history of the Universe, what could help explain older structures.

\section{ACKNOWLEDGMENTS}

The authors would like to thank $\mathrm{CNPq}$ for the financial support that allowed this work to be done. Also, we are grateful for the pertinent suggestions made by the Referee.

[1] A. Sen, Journal of High Energy Physics 2002, 048 (2002).

[2] A. Sen, Journal of High Energy Physics 2002, 065 (2002).

[3] G. Gibbons, Physics Letters B 537, 1 (2002)

[4] T. Padmanabhan, Phys. Rev. D 66, 021301 (2002).

[5] J.-g. Hao and X.-z. Li, Phys. Rev. D 66, 087301 (2002).

[6] J. S. Bagla, H. K. Jassal, and T. Padmanabhan, Phys. Rev. D 67, 063504 (2003)

[7] H. Jassal, Pramana 62, 757 (2004)

[8] L. Abramo and F. Finelli, Physics Letters B 575, 165 (2003)

[9] D.-j. Liu and X.-z. Li, Phys. Rev. D 70, 123504 (2004). 
[10] G. M. Kremer and D. S. M. Alves, General Relativity and Gravitation 36, 2039 (2004).

[11] D. A. Steer and F. Vernizzi, Phys. Rev. D 70, 043527 (2004).

[12] C. Campuzano, S. del Campo, and R. Herrera, Physics Letters B 633, 149 (2006).

[13] R. Herrera, S. del Campo, and C. Campuzano, Journal of Cosmology and Astroparticle Physics 2006, 009 (2006).

[14] H.-H. Xiong and J.-Y. Zhu, Phys. Rev. D 75, 084023 (2007).

[15] L. Balart, S. del Campo, R. Herrera, P. Labraa, and J. Saavedra, Physics Letters B 647, 313 (2007).

[16] M. Sami, P. Chingangbam, and T. Qureshi, Phys. Rev. D 66, 043530 (2002)

[17] V. H. Cárdenas, Phys. Rev. D 73, 103512 (2006)

[18] Y.-S. Piao, Q.-G. Huang, X. Zhang, and Y.-Z. Zhang, Physics Letters B 570, 1 (2003).

[19] S. Srivastava, (2004), arXiv:gr-qc/0409074 [gr-qc].

[20] R. C. de Souza and G. M. Kremer, Classical and Quantum Gravity 26, 135008 (2009)

[21] L. G. Collodel and G. M. Kremer, AIP Conference Proceedings 1647, 29 (2015).

[22] S. Chaplygin, Sci. Mem. Moscow Univ. Math. Phys. 21, 1 (1902).

[23] A. Kamenshchik, U. Moschella, and V. Pasquier, Physics Letters B 511, 265 (2001),

[24] J. Fabris, S. Gonalves, and P. de Souza, General Relativity and Gravitation 34, 53 (2002).

[25] N. Bili, G. Tupper, and R. Viollier, Physics Letters B 535, 17 (2002).

[26] M. C. Bento, O. Bertolami, and A. A. Sen, Phys. Rev. D 66, 043507 (2002).

[27] V. Gorini, A. Kamenshchik, and U. Moschella, Phys. Rev. D 67, 063509 (2003)

[28] G. Kremer, General Relativity and Gravitation 35, 1459 (2003)

[29] A. Frolov, L. Kofman, and A. Starobinsky, Physics Letters B 545, 8 (2002).

[30] V. Gorini, A. Kamenshchik, U. Moschella, and V. Pasquier, Phys. Rev. D 69, 123512 (2004)

[31] L. P. Chimento, Phys. Rev. D 69, 123517 (2004)

[32] S. del Campo and R. Herrera, Physics Letters B 660, 282 (2008).

[33] M. Biesiada, W. Godowski, and M. Szydowski, The Astrophysical Journal 622, 28 (2005)

[34] F.-Y. Wang, Z.-G. Dai, and S. Qi, Research in Astronomy and Astrophysics 9, 547 (2009).

[35] K. Liao, Y. Pan, and Z.-H. Zhu, Research in Astronomy and Astrophysics 13, 159 (2013).

[36] L. Xu and J. Lu, Journal of Cosmology and Astroparticle Physics 2010, 025 (2010)

[37] Y. Wang, D. Wands, L. Xu, J. De-Santiago, and A. Hojjati, Phys. Rev. D 87, 083503 (2013)

[38] G. Gupta, S. Sen, and A. A. Sen, Journal of Cosmology and Astroparticle Physics 2012, 028 (2012)

[39] H. Benaoum, (2002), arXiv:hep-th/0205140 [hep-th]

[40] B. Paul and P. Thakur, Journal of Cosmology and Astroparticle Physics 2013, 052 (2013)

[41] B. Pourhassan and E. Kahya, Results in Physics 4, 101 (2014).

[42] J. Lu, L. Xu, H. Tan, and S. Gao, Phys. Rev. D 89, 063526 (2014)

[43] S. W. Hawking and G. F. R. Ellis, The Large Scale Structure of Space-Time (Cambridge University Press, 1973) cambridge Books Online.

[44] C. L. Bennett, D. Larson, J. L. Weiland, N. Jarosik, G. Hinshaw, N. Odegard, K. M. Smith, R. S. Hill, B. Gold, M. Halpern, E. Komatsu, M. R. Nolta, L. Page, D. N. Spergel, E. Wollack, J. Dunkley, A. Kogut, M. Limon, S. S. Meyer, G. S. Tucker, and E. L. Wright, The Astrophysical Journal Supplement Series 208, 20 (2013)

[45] P. Ade et al. (Planck), Astron. Astrophys 571, A1 (2014), arXiv:1303.5062 [astro-ph.CO].

[46] J. Simon, L. Verde, and R. Jimenez, Phys. Rev. D 71, 123001 (2005).

[47] D. Stern, R. Jimenez, L. Verde, M. Kamionkowski, $\quad$ and S. A. Stanford, Journal of Cosmology and Astroparticle Physics 2010, 008 (2010).

[48] M. Moresco, A. Cimatti, R. Jimenez, L. Pozzetti, G. Zamorani, M. Bolzonella, J. Dunlop, F. Lamareille, M. Mignoli, H. Pearce, P. Rosati, D. Stern, L. Verde, E. Zucca, C. Carollo, T. Contini, J.-P. Kneib, O. L. Fvre, S. Lilly, V. Mainieri, A. Renzini, M. Scodeggio, I. Balestra, R. Gobat, R. McLure, S. Bardelli, A. Bongiorno, K. Caputi, O. Cucciati, S. de la Torre, L. de Ravel, P. Franzetti, B. Garilli, A. Iovino, P. Kampczyk, C. Knobel, K. Kova, J.-F. L. Borgne, V. L. Brun, C. Maier, R. Pell, Y. Peng, E. Perez-Montero, V. Presotto, J. Silverman, M. Tanaka, L. Tasca, L. Tresse, D. Vergani, O. Almaini, L. Barnes, R. Bordoloi, E. Bradshaw, A. Cappi, R. Chuter, M. Cirasuolo, G. Coppa, C. Diener, S. Foucaud, W. Hartley, M. Kamionkowski, A. Koekemoer, C. Lpez-Sanjuan, H. McCracken, P. Nair, P. Oesch, A. Stanford, and N. Welikala, Journal of Cosmology and Astroparticle Physics 2012, 006 (2012)

[49] E. Gaztaaga, A. Cabr, and L. Hui, Monthly Notices of the Royal Astronomical Society 399, 1663 (2009),

[50] C. Zhang, H. Zhang, S. Yuan, S. Liu, T.-J. Zhang, and Y.-C. Sun, Research in Astronomy and Astrophysics 14, 1221 (2014)

[51] Andreon, S., Maughan, B., Trinchieri, G., and Kurk, J., Astron. Astrophys. 507, 147 (2009).

[52] Andreon, S. and Huertas-Company, M., Astron. Astrophys. 526, A11 (2011).

[53] S. del Campo, I. Duran, R. Herrera, and D. Pavón, Phys. Rev. D 86, 083509 (2012). 